\title{
THE TOPOLOGICAL CENTRALIZERS OF TOEPLITZ FLOWS AND THEIR $Z_{2}$-EXTENSIONS
}

\author{
WOJCIECH BUŁATEK AND JAN KWIATKOWSKI
}

\begin{abstract}
The topological centralizers of Toeplitz flows satisfying a condition (Sh) and their $Z_{2}$-extensions are described. Such Toeplitz flows are topologically coalescent. If $\left\{q_{0}, q_{1}, \ldots\right\}$ is a set of all except at least one prime numbers and $I_{0}, I_{1}, \ldots$ are positive integers then the direct sum $\left.\bigoplus_{i=0}^{\infty} Z_{q i}\right|_{i} \oplus Z$ can be the topological centralizer of a Toeplitz flow.
\end{abstract}

\section{Introduction}

In this paper we study the topological centralizers of Toeplitz flows and their $Z_{2}$-extensions. Toeplitz flows are obtained as the orbit closure of special points in $\{0,1\}^{2}$ called Toeplitz sequences. They were introduced by Jacobs and Keane [2]. Many metric and topological properties of Toeplitz flows were investigated by those authors and by Williams [10]. Markley [6], [7] has examined Toeplitz sequences as characteristic sequences over zero-dimensional groups. Lemańczyk [5] used special Toeplitz sequences to produce examples of $Z_{2}$-extensions over dynamical system with discrete spectrum that have Lebesgue component of finite multiplicity. A regular Toeplitz sequence $\omega$ with a period structure $\left\{p_{t}\right\}$, $t \geq 0$, defines a cocycle $\bar{\psi}_{\omega}$ on the group $G$ of $p_{t}$-adic integers. A cocycle $\bar{\psi}_{\omega}$ determines a skew product transformation $T_{\psi}$ on $G \times Z_{2}$. Each transformation $S$ commuting with $T_{\psi}$ can be identified with a pair $\left(T_{g}, f\right)$, where $T_{g}$ is a rotation of $G$ by $g$ and $f$ is a measurable function, $f: G \rightarrow Z_{2}$ [8]. In this case it is natural to say that $T_{g}$ can be lifted to $S \in C\left(T_{\psi}\right)$ (the metric centralizer of $T_{\psi}$ ). The problem how big is the set of such $g$ 's was investigated in [4]. In [3] this set is described for Morse cocycles. But the same problem can be considered from the topological point of view. A dynamical system $(G, m, \widehat{1})$, ( $m$ the Haar measure, $\hat{1}$ the unit element of $G$ ) is metrically isomorphic to $\Theta(\omega)=(O(\omega), \mu, \sigma)$, where $\sigma$ is the shift and $\mu$ the unique $\sigma$-invariant measure [2]. The cocycle $\bar{\psi}_{\omega}$ becomes a continuous cocycle $\psi_{\omega}, \psi_{\omega}: \overline{O(\omega)} \rightarrow Z_{2}[5]$. The latter enables one to define a $Z_{2}$-extension $\widehat{\Theta(\omega)}=\left(0(\omega) \times Z_{2}, \widetilde{\sigma}\right)$ over $\Theta(\omega)$ as a topological dynamical system. Each homeomorphism $\widetilde{S}$ comnuting with $\widetilde{\sigma}$ induces a homeomorphism $S$ commuting with $\sigma$ and $S$ induces a rotation $T_{g}$ 
of $G$. The problem arises to describe the set of those $g \in G$ which can be lifted to an element of $C(\sigma)$ and those $S \in C(\sigma)$ which can be lifted to an element of $C(\tilde{\sigma})$. In this paper we answer these questions assuming that $\omega$ satisfies a condition $(S h)$ (separated holes). Next we construct a class of special Toeplitz flows with the topological centralizers as in the abstract.

\section{Preliminaries}

We summarize some basic definitions and results. We shall use $Z, N$ to denote the integers, the positive integers, respectively. By flow we will mean a pair $(X, T)$, where $X$ is a compact metric space and $T$ is a homeomorphism of $X$ to itself. A flow $(X, T)$ is minimal if $X$ has no proper closed $T$-invariant subset. A flow $(Y, S)$ is a factor of $(X, T)$ if there is a continuous map $\Pi$ of $X$ onto $Y$, with $\Pi \circ T=S \circ \Pi$. If $\Pi$ is a homeonorphism then $(X, T)$ and $(Y, S)$ are isomorphic as flows. Every minimal flow $(X, T)$ has a maximal equicontinuous factor $(G, g), \Pi:(X, T) \longrightarrow(G, g)$, wherc $G$ is a compact metric monothetic group with generator $g$. If $\Pi^{\prime}:(X, T) \longrightarrow\left(G^{\prime}, g^{\prime}\right)$ is any other such factor then there is a factor map $\psi:(G, g) \longrightarrow\left(G^{\prime}, g^{\prime}\right)$ such that $\psi \circ \Pi=\Pi^{\prime}$.

By the topological centralizer of $(X, T)$ we will mean the set of all continuous maps $U: X \longrightarrow X$ which commute with $T$. We use $C(T)$ to denote the centralizer of $T . C(T)$ is automatically a semigroup and it becomes a group if every $U \in C(T)$ is homeomorphism.

Given a finite abelian group $P$, let $\Omega$ be the space of all bisequences over $P$ with its natural compact metric topology and let $\sigma$ be the left shift homeomorphism on $\Omega$. If $\omega \in \Omega$ then $\omega[n]$ will denote the value of $\omega$ at $n \in Z$, and $O(\omega)$ will denote the orbit of $\omega$. A finite sequence $B=(B[0], \ldots, B[n-1])$, $B[i] \in P, n \geq 1$, is called a block. The number $n$ is called the length of $B$ and denoted by $|B|$. If $\omega \in \Omega$ and $B$ is a block then $\omega[i, k], B[i, k]$, $0 \leq i \leq k \leq n-1$, denote the blocks $(\omega[i], \ldots, \omega[k])$ and $(B[i], \ldots, B[k])$ respectively. Let $C=(C[0], \ldots, C[m-1])$ be another block. We say that $B$ appears at the $i-t h$ place in $\omega$ or $C$ if $\omega[i, i+|B|-1]=B$ or $C[i, i+|B|-1]=B$. If $|C|=|B|$ then the sum of $B$ and $C$ is the block $B+C$ such that

$$
B+C=(B[0]+C[0], \ldots, B[n-1]+C[n-1]),
$$

where de symbol " + " is the operation of $P$. Likewise we define a sequence $\left(\omega+\omega^{\prime}\right), \omega^{\prime} \in \Omega$ as

$$
\omega+\omega^{\prime}=\left(\ldots \omega[-1]+\omega^{t}[-1], \omega[0]+\omega^{\prime}[0], \omega[\mathrm{I}]+\omega^{\prime}[1], \ldots\right) .
$$

A $\omega \in \Omega$ is called a Toeplitz sequence if there exists a collection of pairwise disjoint arithmetic progressions $\left\{T_{i}\right\}$ whose union is $Z$ and such that $n, m \in T_{i}$ implies $\omega[n]=\omega[m]$. A Toeplitz sequence $\omega$ is regular if the $T_{i}$ can be choosen so that $\sum_{i} \frac{1}{q_{i}}=1$, where $T_{i}=\left\{r_{i}+k \cdot q_{i} ; k \in Z\right\}$. Let $\overline{O(\omega)}$ be the orbit closure 
of $\omega$. The set $\overline{O(\omega)}$ is a closed, $\sigma$-invariant subset of $\Omega$. By a Toeplitz flow we will mean a pair $(\overline{O(\omega)}, \sigma)=\Theta(\omega)$, where $\omega$ is a Toeplitz sequence.

Assume that $\omega$ is a non-periodic, regular Toeplitz sequence. It is know i2] that a Toeplitz fow $\Theta(\omega)$ is minimal and uniquely ergodic. The maximal equicontinuous factor of $\Theta(\omega)$ was constructed in [10]. We include a part of this construction to introduce ideas we will use later. For $p \in N$ we set

$$
\operatorname{Per}_{p}(\omega)=\left\{n \in N ; \omega[n]=\omega\left[n^{\prime}\right], \text { whenever } n \equiv n^{\prime}(\bmod p)\right\}
$$

By the $p$-skeleton of $\omega$ we will mean a sequence $\omega_{p}$ obtained from $\omega$ by replacing $\omega[n]$ by a new symbol " $-"$ for all $n \notin \operatorname{Per}_{p}(\omega)$. Thus $p$ is a period of $\omega_{p}$. We call $p$ an essential period of $\omega$ if $p$ is the smallest period of $\omega_{p}$. A period structure for $\omega$ is an increasing sequence $\left\{p_{t}\right\}$ of natural numbers satisfying

(a) $p_{t}$ is an essential period of $\omega$, for all $t$,

(b) $p_{t} \mid p_{t+1}$

(c) $\bigcup_{t=0}^{\infty} \operatorname{Per}_{p_{l}}(\omega)=Z$.

Every non-periodic Toeplitz sequence has a period structure.

Jet $G$ be the group of all $p_{i}$-adic integers i.e.

$$
G=\left\{g=\sum_{t \geq 0} g_{t} \cdot p_{t-1} ; \quad 0 \leq g_{t} \leq \lambda_{t}-1\right\}
$$

where $\lambda_{t}=p_{t-1} / p_{t}, t \geq 0$ and $p_{-1}=1$. A $p_{t}$-adic integer $g$ may be represented also as a class of sequences $\left(n_{t}\right), n_{t} \in N$, such that $n_{t+1} \equiv n_{t}\left(\bmod p_{t}\right), t \geq$ 0 . If $\left(n_{t}^{\prime}\right)$ is another sequence satisfying the above condition then $\left(n_{t}\right)$ and $\left(n_{t}^{\prime}\right)$ determine the same $p_{t}$-adic number $g$ iff $n_{t} \equiv n_{t}^{\prime}\left(\bmod p_{t}\right), t \geq 0$. Let $T$ be the translation of $G$ by the unit element $\widetilde{1}$. In $[\mathbf{1 0}]$ it is proved that $(G, T)$ is the maximal equicontinuous factor of $\Theta(\omega)$. To define a corresponding homomorphism $\Pi$ from $(\bar{O}(\omega), \sigma)$ to $(G, T)$ a special partition $\left\{\Pi_{g}\right\}, g \in G$, of $\overrightarrow{O(\omega)}$ was constructed. For fixed $t, t \geq 0$, and $n, 0 \leq n \leq p_{t}-1$, set

$$
\Omega_{n}^{t}=\left\{x \in \overline{O(\omega)} ; x \text { has the same } p_{t}-\text { skeleton as } \sigma^{n}(\omega)\right\}
$$

Then $\Omega_{n}^{t}, n=0,1, \ldots, p_{t}-1$, are pairwise disjoint closed and open subsets of $\overline{O(\omega)}$. For $g \in G, g=\left(n_{t}\right), 0 \leq n_{t} \leq p_{t}-1, n_{t+1} \equiv n_{t}\left(\bmod p_{t}\right)$ we set

$$
\Omega_{g}=\bigcap_{t=0}^{\infty} \Omega_{n_{t}}^{t}
$$

The farnily of sets $\left\{\Omega_{g}\right\}, g \in G$ is partition of $\overline{O(\omega)}$. Each of them is a closed and non-empty set and

$$
\sigma\left(\Omega_{g}\right)=\Omega_{g+\tilde{1}}
$$


(Here the symbol " + "means the operation in $G$. We will use this symbol in different meanings and we will not remark if no confusion becomes). The factor map II : $\overline{O(\omega)} \rightarrow G$ is defined as

$$
\Pi\left(\Omega_{g}\right)=g
$$

The following remark follows easily from the above construction.

Remark 1. If a sequence $\left\{\sigma^{n_{t}}(\omega)\right\}$ is convergent in $\overline{O(\omega)}$ then $\left(n_{t}\right)$ determines a $p_{t}$-adic integer, i.e., for any $t$ there exists $i_{0}$ such that $n_{i} \equiv n_{j}\left(\bmod p_{t}\right)$ whenever $i, j \geq i_{0}$.

Let $A_{t}=\omega_{p_{t}}\left[0, p_{t}-1\right] . A_{t}$ is a block of the length $p_{t}$ with symbols from $P$ and " " (we will call it a "hole"). By a filled place in $A_{t}$ we will mean each place $i$ such that $A_{t}[i] \in P$. A sequence of blocks $\left(A_{t}\right)$ satisfies the following conditions:

(A) The block $A_{t+1}$ is obtained as a concatenation of $A_{t} A_{t} A_{t} \ldots A_{t}$, where some "holes" are filled by symbols of $P$,

(B) $\lim _{t \rightarrow \infty} r_{t} / p_{t}=1$, where $r_{t}$ is the number of the filled places in $A_{t}$ (regularity),

(C) For every $i \in \mathbf{N}$ there exists an index $t$ such that $A_{t}[i] \in P$ and $A_{t}\left[p_{t}-i\right] \in P$.

Converseiy, each sequence of blocks $\left(A_{t}\right)_{0}^{\infty}$ satisfying $(\mathrm{A}),(\mathrm{B})$ and $(\mathrm{C})$ determines a Toeplitz sequence $\omega$ (may be periodic).

In the sequel we change a bit a defnition of a Toeplitz sequence. Suppose that a sequence $\left(A_{t}\right)_{0}^{\infty}$ satisfies the conditions $(A)$ and $(B)$. Then we can define a two-sided sequence $\omega$ in such a way that

$$
\omega\left[i \cdot p_{t},(i+1) p_{t}-1\right]=A_{t},
$$

for all $i \in Z$ and $t \geq 0$. We will call it a $T^{\circ}$-sequence. The sequence $\omega$ can have the symbol " $\left(\bmod p_{t}\right)$, be a $p_{t}$-adic integer. We denote by $A_{t}(g)$ the following block

$$
A_{t}(g)=A_{t} A_{t}\left[n_{t}, p_{t}+n_{t}-1\right] \text {. }
$$

The sequence $\left(A_{t}(g)\right)_{0}^{\infty}$ satisfies the conditions $(\mathrm{A})$ and $(\mathrm{B})$ and hence determines a two-sided sequence $\omega(g)$ given by (2). It is easy to describe the set $G_{0}$ of those $g \in G$ for which $\omega(g)$ is a Toeplitz sequence. Let $G_{2}$ be the set of all $g=\left(n_{t}\right)_{0}^{\infty}$ from $G$ such that $A_{t}\left[n_{t}\right]={ }^{\prime \prime}-"$ for each $t \geq 0$. It follows from (B) that $G_{2}$ is of Haar measure zero. Then the set $G_{1}=G_{2}+Z(Z$ is a subset of $G$ consisting of all elements of the form $k \tilde{l}$, where $k$ is an integer) is of Haar measure zero again. It is not hard to observe that $G_{0}=G-G_{1}$. Now we can define $\overline{O(\omega)}$ as the orbit closure of $\omega$ in the sense that $x=\lim \sigma^{z_{t}}(\omega), z_{t} \rightarrow \infty$, and $x[i] \in P$ for all $i=0, \pm 1, \ldots$ For all $g \in G$ we have $\overline{O(\omega)}=\overline{(\omega(g))}$. If $g \in G_{0}$ then $\omega(g)$ is a Toeplitz sequence what implies that $\Theta(\omega)=(\overline{O(\omega)}, \sigma)$ is 
a Toeplitz flow. We define the sets $\Omega_{g}(\omega), g \in G$, in the same way as above. The construction of the sequences $\omega(h)$ implies

$$
\Omega_{g}(\omega(h))=\Omega_{g+h}(\omega) .
$$

Remark 2. If $\omega$ is a $T^{\circ}$-sequence, then $\omega$ satisfies the property from Remark 1. Therefore $x \in \Omega_{0}(\omega)$ implies that $x$ coincides with $\omega$ at each $i-t h$ place which $\Omega[i] \in P$. Thus if $g \in G_{0}$ then $\Omega_{g}$ is an one-point set and $\Omega_{g}=\{\omega(g)\}$.

Remark 3. For fixed $t \geq 0$ and $0 \leq n \leq p_{t}-1$ set

$$
C_{t}(n)=\left\{g \in G, g=\left(n_{u}\right)_{0}^{\infty} ; n_{t}=n\right\} .
$$

The sets $C_{t}(0), C_{t}(1), \ldots, C_{t}\left(p_{t}-1\right)$ are closed and open subsets of $G$ and

$$
\bigcup_{i=0}^{p_{i}-1} C_{t}(i)=G .
$$

Further we have

$$
C_{t}(0) \stackrel{T}{\rightarrow} C_{t}(1) \stackrel{T}{\longrightarrow} \ldots \stackrel{T}{\longrightarrow} C_{t}(p-1) \stackrel{T}{\rightarrow} C_{t}(0) .
$$

Denote by $\xi_{t}$ a partition of $G$ determined by the family $\left\{C_{t}(i)\right\}, 0 \leq i \leq p_{t}-1$. If $\omega$ is a $T^{\circ}$-sequence then $\omega$ defines a function $\widetilde{\psi}_{\omega}: G \rightarrow P$ such that

$$
\tilde{\psi}_{\omega}(g)=A_{t}[i]
$$

if $g \in C_{t}(i)$ and $A_{t}[i] \in P$. The function $\tilde{\psi}_{\omega}$ is defined on $G$ except of the set $G_{2}$. If $\omega$ is non-periodic then $G_{2}$ is just the set of all $g$ for which $\widetilde{\psi}_{\omega}$ is not continuous. The function $\widetilde{\psi}_{\omega}$ is $\bigvee_{t=0}^{\infty} \xi_{t}$-measurable. Further observe that if $\Pi:(\overline{O(\omega)}, \sigma) \longrightarrow(G, T)$ is the homomorphism defined by (1) then

$$
\psi_{\omega}=\tilde{\psi}_{\omega} \circ \Pi \quad \text { on } \quad \Pi^{-1}\left(G-G_{2}\right),
$$

where $\psi_{\omega}(y)=y[0], y \in \overline{O(\omega)}$.

\section{Minimality of $\widetilde{\Theta(\omega)}$}

Let $\omega$ be the regular non-periodic $T^{\circ}$-sequence over $Z_{2}=\{0,1\}$ with a period structure $\left\{p_{t}\right\}, t \geq 0$. On the one hand $\omega$ determines a Toeplitz fiow $\Theta(\omega)=(\overline{O(\omega)}, \sigma)$. On the other hand $\omega$ determines a $Z_{2}$-extension $\widehat{\Theta(\omega)}$ of $\Theta(\omega)$ defined by

$$
\widetilde{\Theta(\omega)}=\left(\overline{O(\omega)} \times Z_{2}, \widetilde{\sigma}\right)
$$


where

$$
\tilde{\sigma}(y, i)=\left(\sigma(y), i+\psi_{\omega}(y)\right),
$$

$i \in Z_{2}, y \in \overline{O(\omega)}$. Put $X=\overline{O(\omega)} \times Z_{2}$ and denote by $\Pi^{*}$ the natural projection of $X$ on $\overline{O(\omega)}$ i.e.

$$
\Pi^{*}(y, i)=y .
$$

We have the following commutative diagram

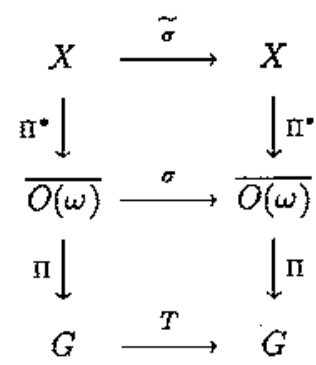

Let $C(\tilde{\sigma})$ and $C(\sigma)$ be the topological centralizers of $\tilde{\sigma}$ and $\sigma$ respectively. If $S \in C(\sigma)$ then $S$ induces a continuous map $S^{\prime}$ on $G$ commuting with $T$ because $(G, T)$ is the maximal equicontinuous factor of $(\overline{O(\omega)}, \sigma)$. But $S^{\prime}$ is a translation by an element $g_{0} \in G$. In this case it is natural to say that $g_{0}$ can be lifted to $S$. The question arises which elcments $g_{0} \in G$ can be lifted to an element of $C(\sigma)$. Notice that if $g_{0} \in G$ can be lifted to $S$, then $S$ is unique because the homomorphism $\Pi$ is one-to-one on $G_{0}$ which is of Haar measure one and the flows $(G, T)$ and $(\overline{O(\omega)}, \sigma)$ are minimal. We will show (proposition 1 below) that if $(X, \widetilde{\sigma})$ is a minimal fiow and $\widetilde{S} \in C(\tilde{\sigma})$ then $\widetilde{S}$ induces an $S \in C(\sigma)$.

The next problem is how to describe those $S \in C(\sigma)$ which can be lifted to elements of $C(\widetilde{\sigma})$. In $\S 3$ we answer these questions provided $\omega$ satisfies additional conditions.

Suppose that a $T^{\circ}$-sequence $\omega$ is determined by a sequence of blocks $\left\{A_{t}\right\}$, $\left|A_{t}\right|=p_{t}$ and each $A_{t}$ is partially filled by 0 's and 1 's. Denote by $k_{t}$ the smallest distance between neighbouring holes in $A_{t}$ i.e. if $A_{t}$ has holes at $I_{1}$-th, $I_{2}$-th, $\ldots, I_{g}$-th places and

$$
I_{1}<I_{2}<\cdots<I_{s}
$$

then

$$
k_{t}=\min \left\{\left[I_{j+1}-I_{j}, \quad j=1,2, \ldots, s-1\right], p_{t}-I_{s}+I_{1}\right\} .
$$

We say that $\omega$ has the property $(S h)$ (separated holes) if

$$
k_{t} \underset{t \rightarrow \infty}{\longrightarrow} \infty \text {. }
$$


Remark 4. If $\omega$ has the property ( $S h$ ) then each $\Omega_{g}, g \in G$, contains at most two points.

In fact, let $g=\left(I_{t}^{\prime}\right) \in G_{2}$ with $0 \leq I_{t}^{\prime} \leq p_{t}-1, I_{t+1}^{\prime} \equiv I_{t}^{\prime}\left(\bmod p_{t}\right)$ and suppose $y, y^{\prime} \in \Omega_{g}$. Then $y\left[-I_{i}^{\prime}, p_{t}-I_{i}^{\prime}-1\right]=y^{\prime}\left[-I_{t}^{\prime}, p_{t}-I_{t}^{\prime}-1\right]=A_{t}$ what implies that the blocks $y\left[-k_{t}, k_{t}\right]$ and $y^{\prime}\left[-k_{t}, k_{t}\right]$ coincide except at the 0 -th place. The condition $k_{t} \rightarrow \infty$ implies that $y$ and $y^{\prime}$ can differ only at the 0 -th place. Simultaneously $\Omega_{g}$ contains precisely two points because $g \in G_{2}$. If $g \in\left(G_{2}+Z\right)$ then the same argument shows that $\Omega_{g}$ contains precisely two points. Of course, if $g \in G_{0}$ then $\Omega_{g}$ consist of the only one point $\omega(g)$.

Proposition 1. If $\widetilde{\Theta(\omega)}$ is a minimal flow and $\widetilde{S} \in C(\widetilde{\sigma})$ then there exist $S \in C(\sigma)$ and a continuous function $\psi: \overline{O(\omega)} \rightarrow Z_{2}$ such that

$$
\widetilde{S}(y, i)=(S(y), i+\psi(y)) .
$$

Moreover the function $\psi$ satisfies a condition

$$
\psi(y)+(S y)[0]=y[0]+\psi(\sigma(y))
$$

Proof: For $a \in G$, a set $\Delta_{a} \subset G \times G$ is defined by

$$
\Delta_{a}=\{(g, g+a) ; g \in G\} .
$$

The sets $\triangle_{a}, a \in G$, are closed, $T \times T$-invariant and minimal. Consider a family of subsets $\widetilde{\Delta}_{a}$ of $X \times X, a \in G$, where

$$
\widetilde{\Delta}_{a}=\left(\Pi^{*}\right)^{-1} \Pi^{-1}\left(\Delta_{a}\right) \quad(\text { see }(3)) .
$$

The sets $\tilde{\Delta}_{a}$ are closed in $X \times X, \tilde{\sigma} \times \tilde{\sigma}$-invariant, pairwise disjoint and

$$
\bigcup_{a \in G} \tilde{\Delta}_{a}=X \times X
$$

Take $\tilde{S} \in C(\widetilde{\sigma})$. The graph $\Gamma$ of $\widetilde{S}$ is a minimal subset of $(X \times X, \widetilde{\sigma} \times \widetilde{\sigma})$ and hence is contained in one of the $\widetilde{\Delta}_{a}^{\prime} s$ i.e.

$$
\tilde{S}\left\{\left(\Omega_{g}, i\right)\right\}=\left\{\left(\Omega_{g+a}, i\right)\right\},
$$

for all $g \in G$ and $i=0,1$. Take $g \in G_{0} \cap\left(G_{0}-a\right)$. Then $(g+a) \in G_{0}$ what means that $\Omega_{g}=\{\omega(g)\}$ and $\Omega_{g+a}=\{\omega(g+a)\}$. Denote $v=\omega(g)$ and $u=\omega(g+a)$. The condition (5) implies

$$
\left\{\begin{array}{l}
\widetilde{S}(v, 0)=(u, \psi(v)) \\
\widetilde{S}(v, 1)=(u, 1+\psi(v))
\end{array}\right.
$$


where $\psi(v)=0$ or 1 .

Now we show that $(6)$ holds for any $y \in \overline{O(\omega)}$. The minimality of the fiow $(X, \tilde{\sigma})$ implies that there exists $r_{n} \rightarrow \infty$ such that

$$
(y, 0)=\lim _{n} \tilde{\sigma}^{r_{n}}(v, 0) .
$$

We have

$$
\tilde{S}(y, 0)=\lim _{n} \widetilde{S} \tilde{\sigma}^{r_{n}}(v, 0)=\lim _{n} \tilde{\sigma}^{r_{n}}(u, \psi(v))=\left(u_{0}, j\right)
$$

and

$$
\tilde{S}(y, 1)=\lim _{n} \tilde{S} \tilde{\sigma}^{r_{n}}(v, 1)=\lim _{n} \tilde{\sigma}^{r_{n}}(u, 1+\psi(v))=\left(u_{0}, 1+j\right),
$$

because $\tilde{\sigma}^{r_{n}}\left(u_{n}, j_{n}^{\prime}\right)=\left(u_{n}, j_{n}^{\prime}\right)$ implies $\widetilde{\sigma}^{r_{n}}\left(u, 1+j^{\prime}\right)=\left(u_{n}, 1+j_{n}^{\prime}\right), j_{,} j^{\prime}, j_{n}^{\prime} \in Z_{2}$, $u_{n}, u_{0} \in \overline{O(\omega)}$.

The last equalities imply (6) for $y$. We can rewrite (6) as

$$
\widetilde{S}(y, i)=(S(y), i+\psi(y))
$$

$i \in Z_{2}$ and $y \in \overline{O(\omega)}$. It is a standard argument that $S \in C(\sigma)$ and $\psi$ is a continuous function. The equality (4) follows from the condition $\widetilde{S} \tilde{\sigma}=\tilde{\sigma} \widetilde{S}$. In this way the proposition is proved.

Proposition 2. If a $T^{\circ}$-sequence $\omega$ satisfies $(S h)$ then $\widetilde{\Theta(\omega)}$ is a minimal flow.

Proof: Suppose $(X, \tilde{\sigma})$ is not minimal. It follows from [9] that there exists a continuous function $f: \overline{O(\omega)} \rightarrow K(K=\{z ;|z|=1\}$ is the circle group) such that

$$
\frac{f(\sigma(y))}{f(y)}=(-1)^{y(0]}
$$

for all $y \in \overline{O(\omega)}$. Thus the function $f^{2}$ satisfies the condition

$$
f^{2}(\sigma(y))=f^{2}(y)
$$

This means that $f^{2}$ is $\sigma$-invariant and hence constant $\left(f^{2}=c\right)$ because $(\overline{O(\omega)}, \sigma)$ is an ergodic system (it is uniquely ergodic). Then the function $\tilde{f}=\frac{1}{c} \cdot f$ satisfes (7) again and $\tilde{f}$ admits only 1 and -1 as its value. So we can assume that $f$ has the above property. Define a function $F: \overline{O(\omega)} \rightarrow Z_{2}$ in the following way

$$
F(y)= \begin{cases}0 & \text { if } f(y)=1 \\ 1 & \text { if } f(y)=-1 .\end{cases}
$$


Then (7) gives

$$
F(\sigma(y))+F(y)=y[0]
$$

for all $y \in \overline{O(\omega)}$.

We will show that (8) implies $\omega$ is a periodic sequence. Without loss of generality we can assume that $\omega$ is a Toeplitz sequence.

As previously let

$$
I_{1}<I_{2}<\cdots<I_{s}, \quad s=s(t)
$$

be places in $A_{t}$ such that $A_{t}\left[I_{j}\right]={ }^{\prime \prime}{ }_{-}^{\prime \prime}, j=1,2, \ldots, s$. First we show that (8) implies $F$ is constant on $\Omega_{1}^{t}$ (see $\S 1$ ) if $I \neq I_{1}, I_{2}, \ldots, I_{\text {, }}$ and $t$ is large enough. There exist a positive integer $L$ such that

$$
F(y)=F\left(y^{\prime}\right)
$$

whenever $y[-L, L]=y^{\prime}[-L, L]$. Take $I$ such that $I_{1}+L<I<I_{2}-L$ (it is possible because $\left.I_{2}(t)-I_{1}(t) \geq k_{t} \rightarrow \infty\right)$. Then $y, y^{\prime} \in \Omega_{1}^{t}$ implies

$$
y[-L, L]=A_{t}\{I-L, I+L]=y^{\prime}[-L, L]
$$

and hence $F(y)=F\left(y^{\prime}\right)$.

Applying (8) we have

$$
F(y)=F\left(y^{\prime}\right)
$$

whenever $y, y^{\prime} \in \Omega_{I}^{t}, I_{1}<I<I_{2}$ because $y[0]=A_{t}[I]=y^{\prime}[0]$. Now we can repeat the above consideration for all $I, 0 \leq I \leq p_{t}-1$ such that $I \neq$ $I_{1}, I_{2}, \ldots, I_{s}$. As a consequence we obtain that $F$ is constant on $\Omega_{I}^{t}$ for $t$ large enough and $I \neq I_{1}, I_{2}, \ldots, I_{s}$.

Denote, by $\widehat{\omega}$ the one-sided sequence obtained from $\omega$ by taking the partial sums of its members in $Z_{2}$ i.e.

$$
\widehat{\omega}=(0, \omega[0], \omega[0]+\omega[1], \omega[0]+\omega[1]+\omega[2], \ldots) .
$$

Set $F\left(\Omega_{0}^{t}\right)=0$ and

$$
i_{j}=F\left(\Omega_{I_{j}+1}^{t}\right), j=1,2, \ldots, s, \quad i_{j} \in Z_{2}
$$

It follows from (8) that

$$
F\left(\Omega_{I_{j}+1}^{t}\right)=i_{j}+\omega\left[I_{j}+1\right]+\cdots+\omega\left[I_{j}+I-1\right]
$$

whenever $2 \leq I<I_{j+1}-I_{j}$, because

$$
y[0]=\omega\left[I_{j}+I\right] \text { if } y \in \Omega_{I_{j}+I}^{t}
$$


Moreover we have

$$
F\left(\Omega_{I}^{t}\right)=\hat{\omega}[I-1]
$$

if $I=1,2, \ldots, I_{1}-1$.

Now suppose that $A_{t+1}\left[I_{j}\right]=0$ or 1 if $j=1,2, \ldots, s$ (it is possible because $\omega$ is a Toeplitz sequence and $i_{1}(t) \rightarrow \infty$ ). We obtain from (9) and (10)

$$
i_{j}=F\left(\Omega_{I_{j}+1}^{t}\right)=F\left(\Omega_{I_{j}+1}^{t+1}\right)=\widehat{\omega}\left[I_{j}\right] .
$$

Thus we can write (9) as

$$
F\left(\Omega_{I}^{t}\right)=\widehat{\omega}[I-1]
$$

whenever $0 \leq I \leq p_{t}$ and $I \neq I_{1}, \ldots, I_{s}$. Using (1I) and the condition $\dot{i}_{1}(t) \rightarrow$ $\infty$ it is not hard to check that $\widehat{\omega}$ is periodic with period $p_{t}$ if $t$ is large enough. Then $\omega$ is a periodic sequence with the same period $p_{t}$ and moreover

$$
\omega[0]+\omega[1]+\cdots+\omega\left[p_{t}-1\right]=0 .
$$

Thus we proved the proposition.

Remark 5. If $S \in C(\sigma)$ can be lifted to an $\widetilde{S} \in C(\tilde{\sigma})$ then it can be lifted to two $\widetilde{S}, \widetilde{S}^{\prime} \in C(\tilde{\sigma})$.

In fact, if

$$
\widetilde{S}(y, i)=(S(y), i+\psi(y))
$$

then the function

$$
\psi^{\prime}(y)=1+\psi(y)
$$

satisfies (4) what implies that $\widetilde{S}^{\prime}$ given by

$$
\tilde{S}^{\prime}(y, i)=\left(S(y), i+\psi^{\prime}(y)\right)
$$

is an element of $C(\tilde{\sigma})$. Suppose that $\widetilde{S}_{1}$ is such that

$$
\widetilde{S}_{1}(y, i)=\left(S(y), i+\psi_{1}(y)\right) .
$$

Then $\psi_{1}$ satisfies

$$
\psi_{\mathrm{I}}(y)+(S y)[0]=y[0]+\psi_{1}(\sigma(y)) .
$$

Adding the above equality and (4) in $Z_{2}$ we obtain

$$
\psi_{1}(y)+\psi(y)=\psi_{1}(\sigma(y))+\psi(\sigma(y)) .
$$

Thus the function $\psi_{1}+\psi$ is $\sigma$-invariant and hence constant (=0 or 1 ) by minimality of $(\overline{O(\omega)}, \sigma)$. So we have

$$
\psi_{1}(y)=\psi(y) \text { or } \psi_{1}(y)=1+\psi(y) .
$$

It follows from (4) that $\widetilde{S}$ is an automorphism iff $S$ is an automorphism. 


\section{The Topological Centralizers of $\Theta(\omega)$ and $\widetilde{\Theta(\omega)}$}

Let $\omega$ be a $T^{\circ}$-sequence satisfying the condition $(S h)$ and as preceding let $G_{2}$ be the set of all $g=\left(I_{t}\right) \in G, 0 \leq I_{t} \leq p_{t}-1, I_{t+1} \equiv I_{t}\left(\bmod p_{t}\right)$ for every $t \geq 0$, and $G_{1}=G_{2}+Z$.

Proposition 3. The flow $(\overline{O(\omega)}, \sigma)$ is topologically coalescent i.e. each $S \in$ $C(\sigma)$ is a homeomorphism.

Proof: Take $S \in C(\sigma)$. By preceding considerations there exists $h=\left(h_{t}\right) \epsilon$ $G, 0 \leq h_{t} \leq p_{t}-1$, such that

$$
S\left(\Omega_{g}\right)=\Omega_{g+h}
$$

for all $g \in G$. If $g \in G_{0}$ then card $\left(\Omega_{g}\right)=1$ and then card $\left(\Omega_{g+h}\right)=1$ so that $\left(G_{0}+h\right) \subset G_{0}$. We will show that $\left(G_{0}+h\right)=G_{0}$.

The map $S$ can be obtained by a code, i.e., there exist integers $k, I$ with $k>0$ and a function $f:\{0,1\}^{k} \rightarrow\{0,1\}$ such that

$$
(S y)[i]=f(y[i+I, \ldots, i+I+k-1]),
$$

for all $i=0, \pm 1, \ldots$ and $y \in \overline{O(\omega)}$. Without loss of generality we can assume that $I=0$. Choose $t$ large enough so that $k_{t}>(2 k+1)$ and consider $\omega^{\prime} \in \Omega_{0}$. Then $S \omega^{\prime} \in \Omega_{k}$.

Suppose that $I_{0}, I_{1}, \ldots, I_{s-1}$ be places in $A_{t}$ such that $A_{t}\left[I_{j}\right]="{ }^{\prime \prime}, j=$ $0,1, \ldots, s-1$. Then $A_{t}$ is of the following form

$$
A_{t}=B_{t}(0) \underline{I_{1}} B_{t}(1) \underline{I_{2}} \ldots \underline{I_{s-1}} B_{t}(s) .
$$

The sequences $\omega_{p_{t}}^{\prime}$ and $\left(S \omega^{\prime}\right)_{p_{t}}$ (see $\S 1$ ) are concatenations of blocks $A_{t}$ and $\left(S \omega^{\prime}\right)_{p_{\mathrm{t}}}$ is the translation of $\omega_{p_{\mathrm{t}}}^{\prime}$ on $h_{t}$. We can compare $\omega_{p_{t}}^{\prime}$ and $\left(S \omega^{\prime}\right)_{p_{\mathrm{t}}}$ using the following figure

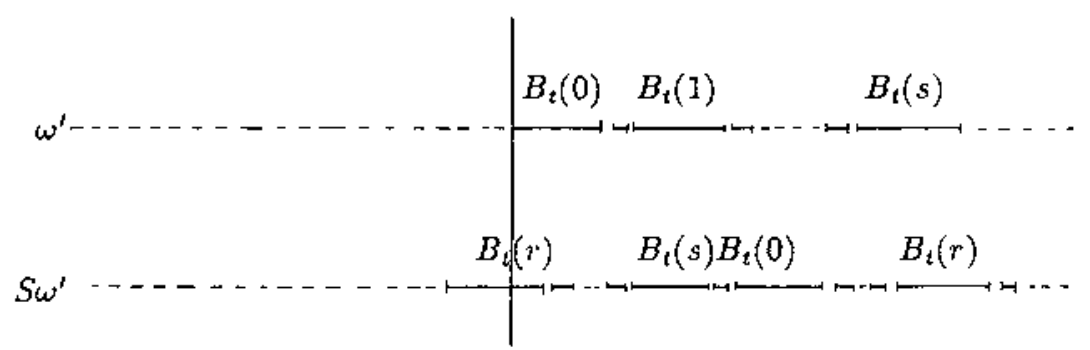

Figure 1.

Using a coding argument it is easy to see that each hole in $\left(S \omega^{\prime}\right)_{p_{t}}$ appears at the place which is distant not more than $k$ places from some hole in $\omega_{p_{i}}^{\prime}$. Thus 
there exists an one-to-one correspondence between the holes in $\omega_{p_{\mathrm{t}}}^{\prime}$ and $\left(S \omega^{\prime}\right)_{p_{\mathrm{t}}}$. This means the following: whenever the block $B_{t}(0)$ $B_{t}(1)$ appears in $\omega_{p_{t}}^{\prime}$ then the block $B_{t}(r) \ldots B_{t}(r+1)$ (see Figure 1) appears in $\left(S \omega^{t}\right)_{p_{t}}$ and they are placed as follows

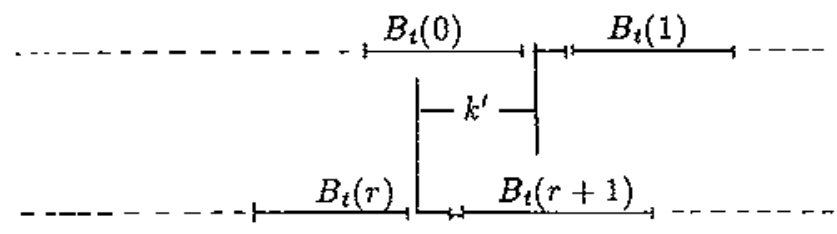

Figure 2.

where $k^{\prime} \leq k$. Both the blocks $B_{t}(0) 0 B_{t}(1)$ and $B_{t}(0) 1 B_{t}(1)$ appear in $\omega^{\prime}$ and the blocks $B_{t}(r) 0 B_{t}(r+1)$ and $B_{t}(r) 1 B_{t}(r+1)$ appear in $S \omega^{\prime}$. Using a coding argument again it is clear that whenever the block $B_{t}(0) 0 B_{t}(1)$ appears in $\omega^{t}$ then $B_{t}(r) b_{0} B_{t}(r+1)$ appears under it which some $b_{0}=0$ or 1 . If $B_{t}(0) 1 B_{t}(1)$ appears in $\omega^{\prime}$ then $B_{t}(r) \tilde{b_{0}} B_{t}(r+1)$ appears in $S \omega^{\prime}\left(\tilde{b_{0}}=1+b_{0}\right)$. We can repeat the same argument for each block $B_{t}(I) 0 B_{t}(I+1)$ and $B_{t}(I) 1 B_{t}(I+1)$, $I=0,1, \ldots, s-1$.

Now, take $g \in G_{2}$. According to Remark 4 there exist precisely two $\omega_{1}, \omega_{2} \in$ $\Omega_{g}$ such that $\omega_{1}[i]=\omega_{2}[i]$ for $i \in Z, i \neq 0$, and $\omega_{1}[0]=\omega_{2}[0]+1$. The above reasoning shows that $S \omega_{1}$ and $S \omega_{2}$ differ at one place (see Figure 2) and they coincide at the remaining places. This means that card $\left(\Omega_{g+h}\right)=2$ so that $g+h \in G_{1}$. The last condition implies $\left(G_{1}+h\right) \subset G_{1}$ and then $G_{1}+h=G_{1}$ because $\left(G_{0}+h\right) \subset G_{0}$. It follows from Remark 4 that $S$ is one-to-one.

Corollary 1. We conclude from the proof of the above proposition that if $h \in G$ can be lifted to an element of $C(\sigma)$, then

$$
\left(G_{1}+h\right)=G_{1}
$$

(equivalently $G_{0}+h=G_{0}$ ), moreover there exists $k>0$ such that

$$
\left(G_{2}+h\right) \subset \bigcup_{I=0}^{k}\left(G_{2}+I\right) .
$$

To answer the question which $h \in G$ satisfying (13) can be lifted to a $S \epsilon$ $C(\sigma)$ we need two notions. By a $t$-symbol of $\omega$ we mean every block $A$ of length $p_{\mathrm{t}}$ such that

$$
A=\omega\left[I p_{t}, I p_{t}+p_{t}-1\right], \quad I=0, \pm 1, \ldots
$$

and all the members of $\omega\left[I p_{t}, I p_{t}+p_{t}-1\right]$ are 0 or 1 . Each $t$-symbol of $\omega$ coincides with $A_{t}$ except at the $I_{0}$-th, $\ldots, I_{s-1}$ th places. The sequence $\omega^{\prime}\left(\omega^{\prime} \in \Omega_{0}\right)$ is a concatenation of $t$-symbols of

$$
\omega^{\prime}=\ldots A_{t}(j-1) A_{t}\left(j_{0}\right) A_{t}\left(j_{1}\right) \ldots
$$


where $A_{t}(\cdot)$ denote $t$-symbols and $A_{t}\left(j_{0}\right)=\omega^{\prime}\left[0, p_{t}-1\right]$. Likewise, if $y \in \Omega_{h}$, $h=\left(h_{t}\right)$, then $y$ is a concatenation of $t$-symbols $A_{t}(h)(\cdot)$ of $\omega(h)$

$$
\begin{gathered}
y=\ldots A_{t}(h)\left(j_{-1}\right) A_{t}(h)\left(j_{0}\right) A_{t}(h)\left(j_{1}\right) \ldots \\
0 \text {-th place }
\end{gathered}
$$

Each of the $t$-symbols of $\omega(h)$ coincides with $A_{t} A_{t}\left[h_{t}, h_{t}+p_{t}-1\right]$ except at the places $I_{0}-h_{t}, \ldots, I_{s-1}-h_{t}\left(\right.$ taken $\left.\bmod p_{t}\right)$.

Define a two-sided sequence $\omega^{t+1}$ as follows

$$
\begin{gathered}
\omega^{t+1}=\ldots \omega\left[I_{0}-p_{t}\right] \ldots \omega\left[I_{s-1}-p_{t}\right] \omega\left[I_{0}\right] \ldots \omega\left[I_{s-1}\right] \omega\left[I_{0}+p_{t}\right] \ldots \omega\left[I_{s-1}+p_{t}\right] \ldots \\
\text { 0-th place }
\end{gathered}
$$

i.e., $\omega^{t+1}$ is the sequence which we should put in the holes of $\omega_{p t}$ to obtain $\omega$. Analogously we define $\omega^{t+1}(h)$.

Theorem 1. An element $h \in G$ satsifying (19) can be lifted to a $S \in C(\sigma)$ if and only if there exists $t$ such that

$$
\omega^{t+1}+\omega^{t+1}(h)
$$

is a periodic sequence with period equal to the number of all holes of $A_{t}$.

Proof: It is casy to prove the necesity repeating the same arguments as in the proof of Proposition 3.

Sufficiency. Suppose that the condition

$$
\begin{array}{r}
\omega^{t+1}+\omega^{t+1}(h)=\ldots B B B \ldots \\
0 \text {-th place }
\end{array}
$$

holds for $t=t_{0}$ with $|B|=s, s$-the number of all the holes in $A_{t}$, and assume

$$
k_{t_{0}}>4 k+1
$$

If (17) is not satisfied then we can take large enough $t>t_{0}$ because (16) is satisfied for each $t \geq t_{0}$.

Using this condition we will construct an one-to-one correspondence $f_{t}\left(\geq t_{0}\right)$ between the sets $Z_{t}$ and $Z_{t}^{t}$ of all the tusymbols of $\omega$ and $\omega(h)$ respectively. Let $t=t_{0}$ again, write $\omega$ and $\omega(h)$ as in (14) and (15) (except at may be one place). Each $t$-symbol of $\omega$ has the form

$$
A_{t}(j)=B_{t}(0) \underline{a_{0}} B_{t}(1) \underline{a_{1}} \ldots \underline{a_{s-1}} B_{t}(s),
$$

where $a_{0}, \ldots, a_{s-1}$ are at the $I_{0}$-th $, \ldots, I_{s-1}$-th places and they depend on $j$. Thus each $t$-symbol is completely determined by its values at the places 
$I_{0}, I_{1}, \ldots, I_{s-1}$. Likewise if $g=\left(g_{t}\right), 0 \leq g_{t} \leq p_{t}-1$, then each $t$-symbol of $\omega(g)$ is determined by its values at the places $I_{0}-g_{t}, I_{1}-g_{t}, \ldots, I_{\mathbf{s}-1}-g_{t}$ (taken mod $p_{t}$ ). The condition (13) implies that each place $I_{0}-h_{t}, \ldots, I_{s-1}-h_{t}$ in $A_{t}(h)$ is distant not more than $k$ places from a place from among $I_{0}, \ldots, I_{s-1}$ in $A_{t}$. That property and (17) define an one-to-one correspondence between the holes in $\omega_{p_{t}}$ and $(\omega(h))_{p_{i}}$. Moreover (16) implies that if a $t$-symbol $A_{t}(j)$ appears in $\omega$ then replacing each $a_{i}, i=0, \ldots, s-1$, (see (18)) by $a_{i}+B[i]$, respectively, we obtain a $t$-symbol $A_{t}(h)(j)$ of $\omega(h)$. It is easy to see that the above operation determines an one-to-one correspondence $f_{t_{0}}$ between the sets $Z_{t_{0}}$ and $Z_{t_{0}}^{t}$. Now we can extend that correspondence between $Z_{t}$ and $Z_{t}^{\prime}$ for $t \geq t_{0}$. Take a $t$-symbol $A_{t}(g)$ of $\omega$. Then $A_{t}($.$) is a concatenation of t_{0}-$ symbols. Then a concatenation of their images by $f_{t_{0}}$ forms a $t$-symbol of $\omega(h)$ (see (16)). In this manner $f_{t}$ is defined. To define $S \in C(\sigma)$ take $y \in \overline{O(\omega)}$ with $y \in \Omega_{g}, g=\left(g_{t}\right)$. Then $y$ is a concatenation of $t_{0}$-symbols

$$
y=\ldots A_{t_{0}}\left(j_{-1}^{\prime}\right) A_{t_{0}}\left(j_{0}^{t}\right) A_{t_{0}}\left(j_{1}^{t}\right) \ldots
$$

with $y\left[-g_{t_{0}}, p_{t_{0}}-g_{t_{0}}-1\right]=A_{t_{0}}\left(j_{0}^{\prime}\right)$. Put

$$
S(y)=\ldots A_{t_{0}}(h)\left(j_{-1}^{\prime}\right) A_{t_{0}}(h)\left(j_{0}^{\prime}\right) A_{t_{0}}(h)\left(j_{1}^{\prime}\right) \ldots,
$$

where

$$
A_{t_{0}}(h)(j)=f_{t}\left(A_{t_{0}}(j)\right), j=j_{0}^{t}, j_{-1}^{t}, j_{1}^{t}, \ldots
$$

It is evident that $S$ is a continuous map commuting with $\sigma$ and $S(y) \in \Omega_{g+h}$. This means that $S$ is a lifting of $h$. Thus the theorem is proved.

Theorem 2. If $\omega$ satisfies the condition $(S h)$ then every $S \in C(\sigma)$ can be lifted to $a, \widetilde{S} \in C(\tilde{\sigma})$.

Proof: Assume that $h=\left(h_{t}\right), 0 \leq h_{t} \leq p_{t}-1$ satisfies the condition of Theorem 1 with $t=t_{0}$ and let $B$ be the corresponding block. We will show (Lemma 1) that we can admit

$$
B[0]+B[1]+\cdots+B[s-1]=0 \quad\left(\text { in } Z_{2}\right) .
$$

Now suppose that (19) holds. Take a $t_{0}$-symbol of the form (18) and construct a $t_{0}$-symbol $A_{t_{0}}(h)(j)$ of $\omega(h)$ as in the proof of Theorem 1. Put $C=A_{t_{0}}(j)+$ $A_{\mathfrak{t}_{0}}(h)(j)$ and denote by $\widehat{C}$ a block obtained from $C$ by taking partial sums of the members of $C$ i.e.

$$
\widehat{C}[i]=C[0]+\cdots+C\{i\} \quad \text { in } Z_{2}, i=0,1, \ldots, p_{t_{0}}-1 .
$$

Now we can define a function $\psi: \overline{O(\omega)} \rightarrow Z_{2}$. For $y \in \overline{O(\omega)}$ with $y \in \Omega_{g}$, $g=\left(g_{t}\right), 0 \leq g_{t} \leq p_{t}-1, g_{t+1} \equiv g_{t}\left(\bmod p_{t}\right)$ define

$$
\Psi(y)=\left\{\begin{array}{ccc}
C\left[g_{t_{0}}-1\right] & \text { if } & g_{t_{0}}>0 \\
0 & \text { if } & g_{t_{0}}=0
\end{array}\right.
$$


We check that $\psi$ satisfies (4). It follows from considerations of the proof of Theorem 1 that

$$
(S y)[0]=y[0]+C\left[g_{t_{0}}\right]
$$

Now using (19), (20) and (21) we can verify (4) in an easy way. To complete the proof it suffices to show (19).

Lemma 1. Let $h=\left(h_{t}\right)$ satisfy the conditions (13) and (16). Then we can find $t_{1}$ satisfying (16) and the corresponding block $B$ satisfying (19).

Proof: It suffices to restrict to the case $h$ is not rational integer. Write again the block $A_{t}\left(t=t_{0}\right)$ in the form

$$
A_{t}=A_{t}(0) \underline{I_{0}} A_{t}(1) \underline{I_{1}} \cdots \underline{I_{s-1}} A_{t}(s)
$$

and let $I_{0}, I_{1}, \ldots, I_{s-1}$ be all places at which $A_{z}$ has holes. If we draw the block $A_{t}(h)=A_{t} A_{t}\left[h_{t}, h_{t}+p_{t}-1\right]$ under $A_{t}$ then the condition(16) says that each hole in it is distant not more than $k$ places from a hole of $A_{t}$. Suppose that the hole of $A_{t}(h)$ with the number $I_{r}, 0 \leq r \leq s-1$ is lying not far from $I_{0}$ in $A_{t}$. Then the $I_{r+1}$ th hole is not far from $I_{1}$ and so on. Let $A_{t+1}^{*}$ be the concatenation of $\lambda_{t+1}$ blocks $A_{t}$

$$
A_{t+1}^{*}=\underbrace{A_{t}(0) \_A_{t}(1) \_A_{t}(s)}_{A_{t}} \cdots \underbrace{A_{t}(0) \_A_{t}(1) \ldots \ldots-A_{t}(s)}_{A_{t}} .
$$

The block $A_{t+1}^{*}$ has $s \lambda_{t+1}$ holes and to obtain a block $A_{t+1}$ we use a block $\alpha^{t+1}$,

$$
\alpha^{t+1}=\alpha^{t+1}(0) \underline{x_{0}} \alpha^{t+1}(1) \underline{x_{1}} \cdots \underline{x}_{s-1}^{t} \alpha^{t+1}\left(s^{t}\right)
$$

by putting the successive members of $\alpha^{t+1}$ in holes of $A_{t+1}^{*}$. We have $\left|\alpha^{t+1}\right|=$ $s \lambda_{t+1}$ and $x_{0}, x_{1}, \ldots, x_{s-1}^{\prime}$ denote the positions in $\alpha^{t+1}$ with holes. If we draw the block $A_{t+1}^{*} A_{t+1}^{*}\left[h_{t+1}, h_{t+1}+p_{t+1}-1\right]$ under $A_{t+1}^{*}$ then there exists exactly one hole in it that appears not far from the first hole in $A_{t+1}^{*}$. That hole determines a place $r^{\prime}$ in $\alpha^{t+1}, 0 \leq r^{\prime} \leq s \lambda_{t+1}-1$. Consider the translation to the left of $\alpha^{t+1}$ by $r^{\prime}$ places. The the block $\alpha^{t+1} \alpha^{t+1}\left[r^{t}, r^{\prime}+s \lambda_{t+1}-1\right]$ is the begining of $\omega^{t+1}(h)$ as well as $\alpha^{t+1}$ is the begining of $\omega^{t+1}$. It is not hard to deduce from (16) that the holes of $\alpha^{t+1} \alpha^{t+1}\left[r^{\prime}, r^{\prime}+s \lambda_{t+1}-1\right]$ appear precisely under the holes of $\alpha^{t+1}$. If we denote by $H_{0}^{\prime}$ the subgroup of $Z_{\lambda_{s}}, \lambda=\lambda_{t+1}$, generated by $r^{\prime}$ then the last property means that the set $\left\{x_{0}, x_{1}, \ldots, x_{s-1}^{\prime}\right\}$ is a sum of cosets of $Z_{\lambda,}$ modulo $H_{0}^{\prime}$. Without loss of generality we can assume that $r^{t}$ is the smallest element of $H_{0}^{\prime}$. Then $r^{\prime} \mid s \lambda_{t+1}$. Replacing $t+1$ by $t+v$ cventually we can assume that there exist successive members of $\alpha^{t+1}$ equal 0 or 1 (because $h_{t} \rightarrow \infty$ ). Suppose that $\alpha^{t+1}[0], \alpha^{t+1}[1], \ldots, \alpha^{t+1}[s-1]$ are 0 or 1 . 
If we write $\tau^{\prime}=q s+u, 0 \leq u \leq s-1$, then $v: r^{\prime} \rightarrow u$ defines a homomorphism of $H_{0}^{\prime}$ to $Z_{s}$. Let $H_{0}=v\left(H_{0}^{\prime}\right)$ and let $c$ the order of $r^{t}$ in $Z_{\lambda s}$. Then we have

$$
\begin{aligned}
& \alpha^{t+1}\left[r^{\prime}\right]=\alpha^{t+1}[0]+B[0], \\
& \alpha^{t+1}\left[2 r^{\prime}\right]=\alpha^{t+1}\left[r^{\prime}\right]+B[u], \\
& \vdots \\
& \alpha^{t+1}\left[(c-1) r^{\prime}\right]=\alpha^{t+1}\left[(c-2) r^{\prime}\right]+B[(c-2) u], \\
& \alpha^{t+1}[0]=\alpha^{t+1}\left[c r^{\prime}\right]=\alpha^{t+1}\left[(c-1) r^{\prime}\right]+B[(c-1) u] .
\end{aligned}
$$

The above equalities give

$$
B[0]+B[u]+\cdots+B[\underbrace{(c-1) u}_{\text {in } Z_{s}}]=0 .
$$

If $\mu=\operatorname{card}\{\operatorname{ker}(v)\}$ then, of course,

$$
B[0]+B[u]+\cdots+B\{(c-1) u]=\mu \cdot \sum_{i \in H_{0}^{\prime}} B[i] .
$$

Taking in (22) the members $\alpha^{t+1}\left[r^{\prime}+j\right], \alpha^{t+1}\left[2 r^{\prime}+j\right], \ldots, \alpha^{t+1}\left[(c-1) r^{\prime}+j\right]$, $j=1,2, \ldots, s-1$, we obtain

$$
B[j]+B[u+j]+\cdots+B[(c-1) u+j]=0 .
$$

Further we have

$$
B[j]+B[u+j]+\cdots+B[(c-1) u+j]=\mu \cdot \sum_{i \in A} B[i],
$$

where $A$ is a coset of $Z_{s}$ modulo $H_{0}^{t}$. In this way we obtain

$$
\mu \cdot \sum_{i=0}^{s-1} B[\hat{i}]=0 .
$$

If $\mu$ is odd then we have $\sum_{i=0}^{s-1} B[i]=0$. If $\mu$ is even then we replace $t+I$ by $t+2$. If $B^{\prime}$ is a block satisfying

$$
\begin{array}{ll}
\omega^{t+2}+\omega^{t+2}(h)=\ldots B^{\prime} & B^{\prime} B^{\prime} \ldots \\
& 0 \text {-th place }
\end{array}
$$

and $\left|B^{\prime}\right|=s^{t}$ (the number of all holes in $A_{t+1}$ ) then it is not hard to deduce that

$$
\sum_{i=0}^{s^{\prime}-1} B^{\prime}\{i]=\mu \cdot \sum_{A \in C}\left(\sum_{i \in A} B[i]\right),
$$

where $A$ is a coset of $Z$, modulo $H_{0}^{\prime}$ and $C$ a set of cosets (some cosets in $C$ can be repeated). So we have

$$
B^{\prime}[0]+B^{\prime}[1]+\cdots+B^{\prime}\left[s^{\prime}-1\right]=0 .
$$

In this way the lemma is proved 


\section{4. $k_{t}$-Toeplitz flows}

In this section we examine a class of Toeplitz flows determined by special $T^{\circ}$-sequences. Given two sequences of positive integers $\mu_{0}, \mu_{1}, \ldots ; s_{0}, s_{1}, \ldots$ such that $\mu_{i}, s_{i} \geq 2,\left(\mu_{i}, \mu_{j}\right)=1$ for $i \neq j$ and $\left(\mu_{i}, s_{j}\right)=1, i, j=0,1, \ldots$, let us denote

$$
\lambda_{t}=\mu_{t} s_{t}, \quad k_{t}=\mu_{0} \cdot \ldots \cdot \mu_{t}, \quad m_{t}=s_{0} \cdot \ldots \cdot s_{t}, \quad p_{t}=k_{t} m_{t} .
$$

We will define a $T^{\circ}$-sequence $\omega$ determined by a sequence of blocks $A_{t}$ with $\left|A_{t}\right|=p_{t}$ in such a way that each $A_{t}$ has $k_{t}$ holes with equal distances between them. To make this precise we defne

$$
A_{t}=\ldots A_{t}(0) \_\cdots A_{t}\left(k_{t}-1\right),
$$

where $A_{t}(0), \ldots, A_{t}\left(k_{t}-1\right)$ are blocks of 0's and 1's with

$$
\left\lfloor A_{t}(j) \mid=m_{t}, \quad j=0,1, \ldots, k_{t}-1 .\right.
$$

In order to obtain a block $A_{t+1}$ we use a block $\alpha^{t+1}$ of a form

$$
\alpha^{t+1}=\chi^{t+1}(0) \_\alpha^{t+1}(1) \_\cdots \alpha^{t+1}\left(k_{t+1}-1\right),
$$

where $1 \_\alpha^{t+1}(j) \mid=s_{t+1}, j=0,1, \ldots, k_{t+1}-1$. The block

$$
\underbrace{A_{t} A_{t} \ldots A_{t}}_{\lambda_{t+1} \text { times }}
$$

has $k_{t} \lambda_{t+1}$ holes and we fill them by using the successive members of $\alpha^{t+1}$. As a consequence we obtain a block $A_{t+1}$ having $k_{t+1}$ holes. If we put

$$
\alpha^{0}=A_{0}
$$

then we can say that the $T^{\circ}$-sequence $\omega$ is determined by a sequence $\left\{\alpha^{t}\right\}_{0}^{\infty}$ of blocks of the form (23). We will call such a $k_{i}$-sequence and corresponding $\Theta(\omega)=(\overline{O(\omega)}, \sigma)$ a $k_{t}$-Toeplitz flow. A sequence $\omega$ is regular because

$$
\frac{k_{t}}{p_{t}}=\frac{1}{m_{t}} \underset{t \rightarrow \infty}{\longrightarrow} 0
$$

To assume $\omega$ is not periodic we will assume that for all $t \geq 0$ and $j ; 0 \leq j \leq$ $k_{t}-1$, there exist at least two places $I, I^{\prime}$ in $\alpha^{2+1}$ such that $\alpha^{t+1}[I]=0$, $\alpha^{t+1}\left[I^{\prime}\right\}=I$ and $I \equiv I^{t} \equiv j\left(\bmod k_{t}\right)$.

Now we describe the topological centralizer of $\Theta(\omega)$. To do this we need to know the set $G_{2}$ of all points in which the corresponding function $\widetilde{\psi}_{\omega}$ is not 
continuous (see Remark 3). Let $Z_{n}$ as usual denote the cyclic group of order $n$. Define a sequence of group homomorphisms

$$
Z_{k_{0}} \stackrel{f_{0}}{\longleftarrow} Z_{k_{1}} \stackrel{f_{2}}{\longleftarrow} Z_{k_{2}} \stackrel{f_{2}}{\longleftarrow} \ldots
$$

such that

$$
f_{t}(i)=i s_{t+1}\left(\bmod k_{i}\right), \quad i \in Z_{k_{t+1}} .
$$

The condition $\left(s_{t+1}, k_{t}\right)=1$ implies that $f_{t}$ is on $Z_{k_{t}}$. Let

$$
C=\left\{\left(j_{t} m_{t}\right)_{t=0}^{\infty} \in G ; \quad 0 \leq j_{t} \leq k_{t}-1 ; \quad j_{t}=f_{t}\left(j_{t+1}\right), t \geq 0\right\}
$$

It is easy to see that $C$ is isomorphic to the group $\Delta$ of $k_{t}$-adic integers numbers and that $G_{2}=C$. So $G_{1}=G_{2}+Z$ is a subgroup of $H$ (not closed) and $G_{1}$ is the direct sum of $C$ and $Z$ because $C \cap Z=\{0\}$. Now we want to describe those $h \in C$ that determine $S \in C(\sigma)$. We have $h+G_{2}=G_{2}$ so $h$ can be lifted to a $S \in C(\sigma)$ iff $\omega^{t+1}+\omega^{t+1}(h)$ is $k_{t}$-periodic sequence for some $t \geq 0$.

Proposition 4. If $h \in C$ and $h$ satisfies (16), then the order of $h$ in $G$ is finite.

Proof: Let $h=\left(j_{t} m_{t}\right) ; 0 \leq j_{t} \leq k_{t}-1 ; f_{t}\left(j_{t+1}\right)=j_{t}$. Then the block $A_{t}(h)$ has the form

$$
A_{t}(h)=A_{t}\left(j_{t}\right) \_A_{t}\left(j_{t}+1\right) \_\cdots A_{t}\left(j_{t}+k_{t}-1\right) .
$$

Therefore the holes in $A_{t}$ and $A_{t}(h)$ appear mod $k_{t}$ precisely at the same places. Assume that the condition (16) holds for $t=t_{0}$. The sequences $\omega$ and $\omega(h)$ are concatenations of the blocks $A_{t}$ and $A_{t}(h)$ and the holes in them are filled by the sequences $\omega^{t+1}$ and $\omega^{t+1}(h)$ respectively. Thus (16) implies that

$$
\begin{array}{ll}
\omega+\omega(h)=\ldots B^{t} & B^{t} B^{t} \ldots \\
& 0 \text {-th place }
\end{array}
$$

where $\left|B^{\prime}\right|=p_{t}$. Denote by $\eta_{0}$ the sequence from the right side of (24). Then we have

$$
S(y)=y+\sigma^{g t} \eta_{0}
$$

where $y \in \overline{O(\omega)}, y \in \Omega_{g}, g=\left(g_{t}\right)_{t=0}^{\infty}, 0 \leq g_{t} \leq p_{t}-1$. Using (25) several times we obtain

$$
S^{g}(y)=y+\sigma^{g_{t}} \eta_{0}+\sigma^{g_{t}+h_{t}} \eta_{0}+\cdots+\sigma^{g_{2}+\{q-1\} h_{\imath}} \eta_{0} .
$$

Let $r$ be the order of $h_{t}$ in $Z_{p_{c}}$. Then we have

$$
\begin{aligned}
& S^{2 r}(y)=y+\sigma^{g t} \eta_{0}+\cdots+\sigma^{g_{t}+(r-1) h_{t}} \eta_{0}+\sigma^{g_{t}+r h_{t}} \eta_{0}+\cdots+
\end{aligned}
$$

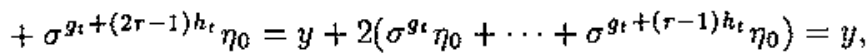


because $r h_{t}=0\left(\right.$ in $\left.Z_{p_{t}}\right)$ and $\sigma^{q} \eta_{0}=\eta_{0}$ whenever $q \equiv 0\left(\bmod p_{t}\right)$ : So we get $S^{2 r}=i d$ and the order of $h$ in $G$ is finite. This finishes the proof.

It is very easy to describe the set of all $h \in C$ with a finite order. Fix $u \geq 0$ and put

$$
\mu_{u}^{t}=\mu_{u+1} \cdot \ldots \cdot \mu_{u+t} \quad \text { for } t \geq 1
$$

Let

$$
H_{u}^{t}=\left\{0, \mu_{u}^{\mathrm{t}}, \ldots,\left(k_{u}-1\right) \mu_{u}^{t}\right\} .
$$

Then $H_{u}^{t}$ is a subgroup of $Z_{k_{\mathrm{s}+t}}$ and the order of $H_{u}^{t}$ is $k_{t}$. Moreover, the homomorphisms $f_{u}, f_{u+1}, \ldots$ in the following sequence

$$
H_{u}^{0}=Z_{k_{u}} \stackrel{f_{u}}{\longleftarrow} H_{u}^{1} \stackrel{f_{u+1}}{\longleftarrow} H_{u}^{2} \stackrel{f_{u+2}}{\longleftarrow} \ldots
$$

are isomorphism. Define

$$
C_{u}=\left\{\left(j_{t} m_{t}\right)_{0}^{\infty} ; 0 \leq j_{T} \leq k_{t}-1, j_{t}=f_{t}\left(j_{t+1}\right), j_{u+v} \in H_{u}^{v}\right\}
$$

We have $C_{u} \subset C_{u+1}, u \geq 0$, and each $C_{u}$ is a subgroup of $C$ with ord $\left(C_{u}\right)=k_{u}$. It is evident that $C^{*}=\bigcup_{u \geq 0} C_{u}$ is a countable subgroup of $C$ and it is the set of all $h \in C$ of a finite order. Thus the topological centralizer of $\Theta(\omega)$ is a subgroup of $C^{*} \oplus Z$. Now we describe a class of $k_{t}$-sequences with topological centralizer equal to $C^{*} \oplus Z$.

Take $0 \leq j \leq k_{t}-1$ and denote by $\alpha_{j}^{t}$ the following block

$$
\alpha_{j}^{t}=\longleftarrow \alpha^{t}(j) \_\alpha^{t}(j+1) \_\cdots \alpha^{t}\left(k_{t}-1+j\right) .
$$

If $h=\left(j_{t} m_{t}\right) \in C$, then the condition (16) can be formulated as follows:

There exists $t_{0} \geq 0$ and a block $B$ with $|B|=k_{t_{0}}$ such that for all $t \geq t_{0}$

$$
\alpha^{t+1}+\alpha_{j_{t+2}}^{t+1}=\underbrace{B_{t} B_{t} \ldots B_{t}}_{\lambda_{t+1}-\text { times }},
$$

where $B_{t_{0}}=B$ and the next blocks $B_{t_{0}+1}, B_{t_{0}+2}, \ldots$ satisfy the recurrent formulas

$$
B_{t+1}[j]=B_{t}\left[f_{t}(j)\right], \quad j=0,1, \ldots, k_{t+1}-1, \quad t>t_{0} .
$$

Now assume additionally that $s_{t+1}>2 k_{t}+1$ for every $t \geq 0$. Take any block $\alpha^{0}$ of a form (23) such that $p_{0}=k_{0} m_{0}$ is the smallest period of the infinite sequence $\alpha^{0} \alpha^{0} \ldots$ Choose blocks

$$
\alpha^{t+1}(0) \_\cdots \alpha^{\alpha^{t+1}}\left(\mu_{t+1}-1\right), \quad t \geq 0,
$$


in such a way that for each $0 \leq j \leq k_{t-1}$ there exist $I \neq I^{\prime}, I \equiv I^{\prime} \equiv j\left(\bmod k_{t}\right)$ with $\alpha^{t+1}(0)[I]=0$, $\alpha^{t+1}(0)\left[I^{\prime}\right]=1$, and

$$
\mu_{t+1} s_{t+1}
$$

is the essential period of (27). Define $\alpha^{2+1}$ of the form (23) by taking the concatenation of $k_{t}$ copies of the blocks (27). As a consequence we obtain a regular $k_{t}$-sequence $\omega$. Moreover the blocks $\left\{\alpha^{t}\right\}$ satisfy (26) if $j_{t+1}$ is the multiplicity of $\mu_{t+1}$ and $B_{t}=00 \ldots 0$. By easy considerations we can prove that every $h \in C^{*}$ can be lifted to a $S \in C(\sigma)$. Therefore $C(\sigma)=C^{*} \oplus Z$.

It remains to prove that the numbers $\left\{p_{t}\right\}, t \geq 0$, form the period structure of $\omega$, i.e., $p_{t}$ is the essential period of $\omega_{p_{t}}$ for every $t \geq 0$. It is true if $t=0$ by our choice of $\alpha^{0}$. Suppose that $p_{t}$ is the smallest period of $\omega_{p_{t}}$. We will show that $p_{t+1}$ is the essential period of $\omega_{p_{t+1}}$. Then the smallest period $p^{t}$ of $\omega_{p_{t+1}}$ is the muitiplicity of $m_{t+1}$ because $\omega_{p_{t+1}}$ has the holes every $m_{t+1}$ places starting from 0 -th place. On the other hand $p^{\prime}$ is the multiplicity of $p_{t}$. In fact, if

$$
p^{\prime}=I \cdot p_{t}+r, \quad 0 \leq r \leq p_{t}-1,
$$

then $p^{\prime}$ is a period of $\omega_{p_{t}}$ so $r$ is. The condition $r<p_{t}$ implies $r=0$. We have shown that $p^{\prime}$ is the multiplicity of the smallest common multiplicity of $p_{t}$ and $m_{t+1}$. We have

$$
\left[p_{t}, m_{t+1}\right]=m_{t}\left[k_{t}, s_{t+1}\right]=m_{t} k_{t} s_{t+1}, \text { because }\left(k_{t}, s_{t+1}\right)=1 .
$$

At the same time it is easy to deduce that the assumption (28) implies $p^{\prime}$ is the multiplicity of $\mu_{t+1} s_{t+1} m_{t}$. Now the condition $\left(k_{t}, \mu_{t+1}\right)=1$ implies $p^{\prime}=s_{t+1} m_{t} k_{t} \mu_{t+1}=m_{t+1} k_{t+1}=p_{t+1}$. In this manner the sequence $\left\{p_{t}\right\}_{0}^{\infty}$ is a period structure of $\omega$.

Let $q$ be a fixed prime number and $q_{0}, q_{1}, \ldots$ all the remaining prime numbers. We can admit $s_{t}=q^{t+1}$ and $\mu_{t}=q_{t}^{J_{t}}$ for $t \geq 0$, where $I_{t}$ are positive integers. Here the group $C^{*}$ is isomorphic to $\oplus_{0}^{\infty} Z_{\mu_{t}}$ the direct sum of the cyclic groups $Z_{\mu_{t}}$. Thus the group $\oplus_{0} z_{\mu_{t}} \oplus Z$ can be the topological centralizer of a Toeplitz flow.

\section{References}

1. G. Christol, T. Kamae, M. Mendes-France, G. Rauzy, Suites algebrajques automates et substitutions, Bull. Soc. Mat. France 108 (1980), 401-409.

2. K. JaCobS, M. KEANE, Sequences of Toeplitz type, $Z$. Waht. verw Geb. $13(1969), 123-131$.

3. J. KWIATKOWSKI, T. ROJEK, The centralizer of Morse shifts induced by arbitrary blocks, Studia Math. 88 (to appear). 
4. J. KWIATKOWSKI, M. LEMAŃCZYK, The centralizer of ergodic group extensions, Proceedings of Warsaw semestre on Ergodic Theory (1986) (to appear).

5. M. LemańcZYK, Toeplitz $Z_{2}$-extensions, Ann. $I$. H. Poincare (to appear).

6. N.G. MARKLEY, Characteristic sequences, Z. Wahr. verw Geb. 30 (1974), 321-342.

7. N.G. MAFKLEY, Substitution-like minimal sets, Israel Jour. Math. 22 (1975), 332--353.

8. D. NEwTON, On canonical factors of ergodic dynamical systems, J. London Math. Soc. 2, 19 (1979), 129-138.

9. W. PARRY, Compact abelian group extensions of discrete dynamical systems I, Z. Wahr. verw Geb. 13 (1969), 95-133.

10. S. Williams, Toeplitz minimal flows which are not uniquely ergodic, $Z$. Wahr. verw Geb. 67 (1984), 95-107.

\author{
Uniwersytet Mikolaja Kopernika \\ Instytut Matematyki \\ Ul. Chopina 12-18 \\ 87-100 Torun \\ POLAND
}

Rebut el 10 d'Octubre de 1988 